\title{
Erratum to: Omega-6 docosapentaenoic acid-derived resolvins and 17-hydroxydocosahexaenoic acid modulate macrophage function and alleviate experimental colitis
}

Cheng-Ying Chiu - Beate Gomolka Cordula Dierkes Nora R. Huang • Maik Schroeder • Martin Purschke • Dieter Manstein - Bindi Dangi - Karsten H. Weylandt

(C) Springer Basel AG 2012

Erratum to: Inflamm. Res. (2012) 61:967-976

DOI 10.1007/s00011-012-0489-8

In the original publication of the article, the author contribution statement has been missed. The statement should read as given below.

The first two authors Cheng-Ying Chiu and Beate Gomolka contributed equally to this work.

The online version of the original article can be found under doi:10.1007/s00011-012-0489-8.

C.-Y. Chiu - B. Gomolka - C. Dierkes - N. R. Huang ·

M. Schroeder · K. H. Weylandt

Department of Hepatology, Gastroenterology and

Endocrinology, Charité University Medicine Berlin,

Campus Virchow Klinikum, Berlin, Germany

C.-Y. Chiu - N. R. Huang - K. H. Weylandt

Laboratory for Lipid Medicine and Technology, Massachusetts

General Hospital, Harvard Medical School, Boston, MA, USA

M. Purschke · D. Manstein

Wellman Center of Photomedicine, Massachusetts General

Hospital, Harvard Medical School, Boston, MA, USA

B. Dangi

DSM Nutritional Products, Columbia, MD, USA

K. H. Weylandt ( $\square)$

Med. Klinik m. S. Gastroenterologie and Hepatologie,

Charité Campus Virchow Hospital, Augustenburger Platz 1,

Berlin 13353, Germany

e-mail: karsten.weylandt@charite.de 